\title{
ACCIÓN COLECTIVA Y COOPERATIVISMO EN LA AGRICULTURA CUBANA
}

\author{
HANS-JÜRGEN BURCHARDT
}

Universidad de Hannover. Alemania.

\section{PALABRAS CLAVE ADICIONALES}

Cooperativas agrarias, Socialismo cubano, Cambio social, Latinoamérica.

\author{
ADDITIONAL KEYWORDS \\ Agricultural cooperatives, Cuban socialism, \\ Social change, Latin America.
}

RESUMEN. A partir de la información recogida en una investigación realizada entre universidades alemanas y cubanas, se analiza en este artículo la introducción del modelo cooperativista en la agricultura cubana en 1993, y se hace balance del desarrollo de las UBPC (unidades básicas de producción cooperativa). En primer lugar, analiza los problemas estructurales de la agricultura cubana y la dependencia alimentaria heredada de la época anterior a la revolución castrista. En segundo lugar, analiza las causas del fracaso del Programa Alimentario de 1989, como precedente de las reformas introducidas en 1993 en lo que algunos autores han denominado la "nueva reforma agraria". Finalmente, el autor expone una serie de reflexiones personales sobre las posibilidades de consolidación de estas nuevas formas de cooperativismo en Cuba, y plantea las actuaciones de política agraria que serian necesarias para que las UBPC se conviertan en un elemento fundamental del desarrollo rural en Cuba.

SUMMARY. On the base of a research project of collaboration between German and Cuban universities, this article analyzes the implementation of cooperativist models in 1993 and the later development of the UBPC (basic unities of cooperative production) in the Cuban agriculture. Firstly, his author analyses the structural problems of the Cuban farming sector and the food dependency of Cuba as a heritage received by the Castrist revolution. Secondly, he analyses the unsuccessful 1989 Food Program, seeing there the reasons of the reforms implemented in 1993 in the farming sector, which are called by some authors "the new agricultural reform". Finally, the author offers some personal reflections on the posibilities of consolidations of the UBPCs in Cuba, and he poses the need of a new agricultural policy that supports this new form of cooperativism as fundamental factor of the development of the Cuban rural society .

E-mail: burchard@vossnet.de

\section{Revista Internacional de Sociología (RIS)}

Tercera Época, $n^{\circ}$ 28, Enero-Abril, 2001, pp. 243-258. 


\section{R IS}

La solución de la problemática agraria será para algunos países de América Latina uno de los retos centrales del siglo XXI. En Cuba se experimenta una respuesta a este desafio a través de una reforma sin precedentes. A partir de 1993 se transformó casi toda la agricultura estatal en cooperativas autogestionadas. Sobre estos cambios agrarios se terminó recientemente una investigación, que tuvo más de tres años de duración, mediante una fructífera cooperación entre la Universidad de Hannover, Alemania, con dos centros científicos de la Universidad de La Habana'. El objetivo de la investigación fue analizar las potencialidades, obstáculos e implicaciones socioeconómicas de estas transformaciones; identificar sus actores sociales, así como revelar las nuevas posibilidades creadas para el desarrollo y las limitaciones que inhiben dichos cambios. El texto siguiente presenta algunos de los resultados arrojados por la investigación ${ }^{2}$.

\section{ANTECEDENTES}

Entre mediados del siglo XVIII y XIX, con el desarrollo de la economía de plantación, se consolidó en Cuba un modelo económico basado en la exportación de productos primarios, los cuales dejaron una impronta que, aún hoy día, en sus líneas más gruesas persisten no sólo en la Isla, sino en muchas zonas de la región. Las exportaciones de tabaco, azúcar y café, así como el comercio de esclavos, fueron, al mismo tiempo, por más de 150 años, renglones principales en los intercambios del mercado mundial. En esta etapa de la época colonial se formó un nuevo modelo de acumulación, que fue, a la vez, agrario y semi-industrial.

Como rasgo fundamental, la economía de plantación padecía de una escasez crónica de fuerza de trabajo en el campo, originada por la ausencia de mano de obra libre y por su alto valor, así como por la inexistencia de una gran masa de pequeños propietarios. La solución a la falta de brazos la resolvió la introducción masiva de esclavos. Otro rasgo central del modelo lo constituyó la importación creciente de alimentos y bienes de uso duraderos, que no podían ser cubiertos por la producción propia, y que se reflejó en un déficit histórico con respecto a la demanda de alimentos. Cuba se convertiría en una economía agraria, pero no campesina.

\footnotetext{
' Las instituciones que colaboraron en el proyecto fueron el Departamento de Historia de la Universidad de Hannover, el Equipo de Estudios Rurales (EER) y el Centro de Estudios de la Economia Cubana (CEEC) de la Universidad de La Habana.

${ }^{2}$ El artículo refleja los resultados del proyecto de investigación "La transformación de la agricultura cubana a partir de 1993", dirigido por el autor y auspiciado por la Fundación Volkswagen.
} 


\begin{tabular}{|c|c|}
\hline & REIISTA INTERNaCIONAL DE SOCIOLOCiA \\
\hline ICCIÓN COLECTIVA Y COOPERATIVISMO EN LA AGRICULTURA CUBANA & No 28. Enero-Abril, 20 \\
\hline
\end{tabular}

Por otra parte, la economía cubana no encontró solución en la etapa republicana a la existencia del latifundio agrario como fenómeno económico, que fue agravado por la presencia de los monopolios y capitales venidos de Estados Unidos. Esta herencia, transferida a la Revolución triunfante en enero de 1959, seguiría teniendo como puntos nodales la problemática de la tierra y la abundancia de fuerza de trabajo, ahora excedente durante largas épocas del año, así como la dependencia externa en bienes alimentarios y en casi todos los demás géneros de uso duradero. Las reformas agrarias de 1959 y 1963 llevaron, de un lado, al predominio estatal en la explotación de la tierra, y de otro, introdujeron modelos para la producción en gran escala y, sobre todo, para aprovechar la infraestructura existente dando prioridad a la producción de azúcar. Bajo el lema más propiedad estatal, más socialismo, a partir de 1963 más del $70 \%$ del sector agropecuario fue estatalizado y convertidos en asalariados la gran mayoría de los trabajadores allí ocupados (Arandẳ, 1968; Valdés, 1990).

Un hecho significativo en las reformas agrarias realizadas por la Revolución lo constituyó la especificidad de no haber repartido tierra, sino en haber convertido en propietarios a los arrendatarios, aparceros y precaristas (Pino, 1999). En otras palabras, al latifundismo colonial y "neocolonial" le siguió, casi ininterrumpidamente, un latifundismo estatal que por supuesto se distinguió de sus antecedentes por su particularidades (Valdés, 1997) y, sobre todo, por su carácter social, pero caracterizándose también por seguir una lógica de racionalización fordista: "Su consecuencia inmediata fue la ampliación y reforzamiento del modelo agrícola de altos insumos en unidades productivas cada vez más gigantes para la producción en masa y bajo un fuerte régimen verticalizado de dirección" (Figueroa, 1996: 11).

Tabla 1.

Tamaño promedio de las empresas agropecuarias estatales 1990.

\begin{tabular}{lc}
\hline Tipo de empresa & Tamaño promedio (hectáreas) \\
\hline Complejos Agro-Industriales (CAI) Azucareros & 13.110 \\
Cultivos varios & 4.276 \\
Cítricos y frutales & 10.822 \\
Arroz & 32.760 \\
Tabaco & 2.778 \\
Pecuario (ganadería) & 24.865 \\
\hline
\end{tabular}

Fuente: NOVA, 1997, pp. 36. 


\section{RIS}

Este latifundio estatal se orientaba al uso masivo de la mecanización, introducción de la ciencia y técnica, quimización, especialización productiva, etc., además de promover la constitución de grandes explotaciones agropecuarias. Los crecimientos de la producción en estas condiciones se obtenían mediante la concentración de maquinaria, equipos, altos insumos, etc., acompañados de una escasez de fuerza de trabajo, suplida con la movilización masiva de trabajadores de la ciudad.

En otras palabras, la producción agraria cubana tomó un carácter típicamente extensivo. Entre sus deficiencias más significativas pueden enumerarse, entre otras, el que los aumentos de la producción no se correspondían con el aumento de las inversiones en insumos, obras de infraestructura, ciencia y técnica empleada, etc.; los rendimientos y la productividad del trabajo mostraban una permanente regresión. La escasa rentabilidad de las empresas estatales se convirtió en un pesado lastre para el presupuesto del Estado, que subvencionaba las pérdidas; en general, la agricultura no aportó saldos productivos suficientes como para alcanzar una base alimentaria que facilitara una relativa independencia en la satisfacción de los rubros principales de la alimentación de la población.

Tabla 2.

Indicadores globales del sector agropecuario (en millones de pesos).

\begin{tabular}{lccccc}
\hline Años & $\begin{array}{c}\text { Rendimientos de } \\
\text { los fondos } \\
\text { básicos }\end{array}$ & $\begin{array}{c}\text { Producto } \\
\text { capital }\end{array}$ & $\begin{array}{c}\text { Inversión por } \\
\text { peso producido }\end{array}$ & $\begin{array}{c}\text { Dotación fondo } \\
\text { básico por } \\
\text { trabajador }\end{array}$ & $\begin{array}{c}\text { Consumo } \\
\text { producto por } \\
\text { producción bruta }\end{array}$ \\
\hline 1975 & 2,21 & 4,50 & 0,22 & 2.480 & 0,55 \\
$1976-80$ & 1,25 & 5,54 & 0,18 & 3.573 & 0,56 \\
$1981-85$ & 0,76 & 4,06 & 0,25 & 7.645 & 0,63 \\
$1986-89$ & 0,61 & 3,88 & 0,26 & 10.002 & 0,68 \\
\hline
\end{tabular}

Fuente: NOVA, 1997, p. 40.

Por otra parte, al incorporarse Cuba a la división internacional socialista del trabajo a partir de 1972, asume la especialización en la producción de azúcar y cítricos a cambio de cereales y otros bienes alimenticios. A fin de los años 80 , cerca del $60 \%$ del área cultivable se destinaba a los productos exportables y algo más del $40 \%$ a la producción de alimentos. Esto arrojaba aproximadamente un 0,14 ha de superficie cultivada per-cápita para la manutención. Al no lograrse los 
objetivos trazados en la especialización, Cuba se convirtió en un país dependiente de las importaciones para satisfacer las necesidades de la población. Así, en los inicios de la década de los 90 , el $55 \%$ de las calorías, el $50 \%$ de las proteínas y el $90 \%$ de todas las grasas consumidas en la Isla provenían del exterior (Figueras, 1994). Esta dependencia, hasta hoy día, en términos generales se mantiene.

Con el derrumbe del socialismo en Europa de Este y con el objetivo de sustituir con producciones propias el déficit de los productos alimenticios que provenían de este área, se instrumentó el llamado programa alimentario (1989). El objetivo central de esta política fue reducir la dependencia de las importaciones de alimentos, aumentar el nivel de autosuficiencia y garantizar a la población el suministro adecuado de calorías y proteínas. El conjunto de soluciones apuntadas no modificó, en sus elementos esenciales, las bases, estructuras y condiciones de funcionamiento de la gestión y dirección de la economía agraria cubana. La organización sectorial, la subordinación vertical, la centralización de planes y programas, etc., mantuvieron su vigencia, e incluso se expandieron, abarcando el sector estatal un $83 \%$ de la superficie cultivable (Figueroa, 1996).

Sin embargo, los ambiciosos objetivos del programa alimentario no se realizaron. Las superficies previstas a poner en explotación no fue posible alcanzarlas al reducirse drásticamente los recursos necesarios. Fuentes oficiales reconocieron que, a partir de 1992, se habían reducido los insumos a una quinta parte de los antes utilizados. Al mismo tiempo, se hicieron acuciantes los déficit de fuerza de trabajo, que trataron de compensarse con movilizaciones masivas de la población; mientras tanto se manifestaron deficiencias en la distribución de los productos cosechados, llegando a reconocerse en diferentes medios de prensa que sólo la red comercial recibía un tercio de lo obtenido, una tercera parte se consumía en el campo y otro tercio iba al mercado negro. Estos resultados convirtieron a la agricultura en el mayor sector subvencionado por el presupuesto estatal: "Con el Plan Alimentario fracasó el pilar básico de la estrategia económica estatal en el área de la economía interna" (Mesa-Lago, 1995: 62).

El descalabro del programa alimentario provocó una crisis de los principales sectores de abastecimiento agropecuario, que no pudo ser totalmente amortiguada a través de las importaciones. Las raciones garantizadas por la Libreta de Abastecimiento se redujeron al mínimo. La población sólo pudo escapar de la escasez de alimentos acudiendo al mercado negro, el cual, a su vez, creció tan rápidamente como la inconformidad de los cubanos (Burchadt, 1995; González, 1995). El agudizamiento de la situación alimenticia sólo podía ser superada mediante un cambio estructural en la agricultura. Había llegado el momento de plantearse lo que algunos especialistas llamaron una "nueva reforma agraria". 
RIS

RETISTA INTERNACIONAL DE SOCIOLOCII

Na 28. Enero-Abril. 2001

HANS-JÜRGEN BURCHARDT

\section{COOPERATIVISMO SIN COOPERATIVAS}

El 15 de septiembre de 1993 se abrió un nuevo capítulo en la historia agraria de la Isla. La propuesta del Buró Politico del Comité Central del Partido Comunista de Cuba, posteriormente legislada, inició un proceso de reestructuración del entramado agropecuario del país. Dentro de los predios de las 735 granjas estatales cañeras y las 835 empresas agropecuarias, se crearon las Unidades Básicas de Producción Cooperativa (UBPC).

El Decreto Ley 142 de septiembre del 1993 dispuso la entrega de una parte fundamental de las tierras estatales, en usufructo gratuito, a los colectivos obreros del sector agropecuario, así como traspasarle los medios básicos necesarios para que asumieran sus responsabilidades productivas. Los propósitos con la creación de las UBPC estaban encaminados a lograr incrementos substanciales en las producciones agropecuarias, reducir los costos, estimular la incorporación a las labores agrícolas de nuevos productores, contribuir a la superación de la inestabilidad de la fuerza de trabajo, mejorar los niveles de vida en el agro, facilitar la solución de problemas, como el de la vivienda, y extender los servicios sociales, así como favorecer la eliminación de las subvenciones a la agricultura.

Por otra parte, las UBPC aportaban una reducción significativa del tamaño de las explotaciones agropecuarias al promediar extensiones entre 800 y 1.000 hectáreas por unidad productiva, lo que, unido a la existencia de otras formas de explotación agraria, llevó a que en la Isla pudiera hablarse del surgimiento de una economía mixta en el sector rural. El sector estatal apenas retiene hoy el $33 \%$ de la superficie cultivada, mientras las UBPC concentran el " $42 \%$ de las tierras, el $90 \%$ de la producción cañera y el $60 \%$ de la no cañera" (Valdés, 1997: 185).

Los miembros de la UBPC organizan el trabajo, deciden sobre el empleo de los medios a su disposición, disponen de áreas de autoabastecimiento y comercializan los excedentes de las producciones una vez cumplidos los contratos firmados. Sus planes productivos se fijan en convenios con las empresas estatales, dentro de las cuales funcionan, compran y venden sus productos según los precios determinados por el Estado. Hay analistas que afirman que, con estas transformaciones, "...el gigantesco sector de la granja estatal fue, en realidad, privatizado" (Deere, 1994: 3). No obstante, el Estado se ha reservado el derecho a "...la disolución de cualquier Unidad Básica de Producción Cooperativa... por causas de interés económico o social determinado por el Gobierno" (Gaceta Oficial, 1993).

Sin embargo, las UBPC aportan una democratización de las estructuras organizativas de la producción agropecuaria, pues cada junta directiva de la UBPC tiene que ser confirmada regularmente en elecciones con la asistencia de un $75 \%$ de todos los miembros. Ellas, en opinión de muchos analistas, pueden 


\section{RIS}

ha subrayado que constituyen, en realidad, una entidad dual a medio camino entre la empresa estatal y la verdadera cooperativa; lo que definiríamos como dualismo estructural. De otro lado, se encuentran a medio camino entre una empresa comercial y una simple unidad técnico-productiva, lo que podríamos denominar dualismo funcional. En este desdoblamiento radica, en mi opinión, la complejidad actual de la cuestión agraria en Cuba y la incoherencia de las nuevas estructuras implementadas.

Precisamente, un ángulo que ha hecho evidente este dualismo corresponde a los rendimientos económicos que han mostrado las UBPC. En casi una década de existencia, aún no resultan suficientes los dividendos que arrojan estas cooperativas de nuevo tipo. Sin embargo, si bien se reconoce que, entre 1994-1999, según datos estimados, las UBPC cañeras y de cultivos varios recibieron una ayuda estatal que supera los 1.300 millones de Pesos, para el año 2000 el apoyo del Estado se reduce a cerca de 50 millones (Millares, 1999), lo que constituye un paso alentador.

De acuerdo con los resultados de varios estudios multidisciplinarios (EER, 1996; 1998), las dificultades que presentan las transformaciones habidas en el sector agrario son de diversa naturaleza, y pueden ser desglosadas en tres dimensiones: coyunturales, socioculturales y estructurales.

Por coyuntural se entiende las trabas que causaron el cambio de un modo de producción mecanizado y administrativo por otro de trabajo intensivo y autogestionado, y que, necesariamente, requiere un proceso de consolidación y experiencia de varios años. Aquí pueden mencionarse, entre otros, la falta de conocimientos empresariales, el proceso gradual de asimilación de nuevas tecnologías, el empleo de técnicas intensivas de trabajo, la institucionalización de renovados esquemas organizativos de gestión, el perfeccionamiento y la estabilidad de las juntas directivas, etc. No obstante, las experiencias obtenidas señalan que muchas de estas limitaciones están siendo superadas y algunas de sus lecciones pueden ser generalizadas.

Los problemas socioculturales se identifican con las pautas de comportamiento de los actores implicados en el trabajo de las UBPC. Este movimiento cooperativo surgió de una directiva gubernamental, orientado desde arriba y sin contar con un desarrollo orgánico y evolutivo desde la base. Ambos elementos se reflejan en el lento cambio de conducta de los participantes en el proceso de consolidación de las relaciones que las nuevas entidades demandan.

Este conjunto de circunstancias contribuyó a la estructuración de un extendido paternalismo, aún no totalmente erradicado por parte de la administración estatal, al no reconocerle la autonomía indispensable a estas cooperativas. Tampoco puede ignorarse que persiste un cierto acomodamiento en las direcciones de las UBPC para aceptar el control y la injerencia que ejercen las instancias estatales. Con razón se ha destacado que "uno de los problemas más serios ha sido la imposibilidad de cambiar con rapidez la mentalidad de los agentes involucrados 
en el funcionamiento de las UBPC, tanto de los productores asociados en éstas, como de los funcionarios estatales. Los últimos no renuncian fácilmente a ejercer las prerrogativas y funciones que les otorgaba el esquema administrativo anterior, en tanto los primeros no se sienten dueños de lo que producen" (Carranza, Gutiérrez y Monreal, 1995: 46).

El aspecto sociocultural implica a las relaciones interempresariales. Ante todo, cabe resaltar que Cuba carecía de una gran masa de pequeños productores agrícolas; la gran mayoría de los ocupados en las labores agrarias eran asalariados con una especialización y participación en la división del trabajo relativamente alta. De aquí que, los miembros de las UBPC, no disponían de una cultura autogestionaria, ni habían tenido tiempo para desarrollar una conciencia capaz de asimilar los modos de producción e intensidad de trabajo que requiere la organización cooperativa. Por otra parte, es numeroso la cantidad de miembros de las UBPC que aún no perciben los vínculos entre sus expectativas personales y los resultados económicos de la cooperativa; otros consideran más atractivo mantenerse como asalariados o como pequeños productores independientes; también es frecuente la ausencia de criterios de racionalidad económica. En otras palabras, los miembros de las cooperativas continúan sintiéndose muchas veces como asalariados y no como dueños verdaderos de la producción. Tampoco puede ignorarse que el alto grado de urbanización de la Isla y lo poco atractivo de las condiciones socio-culturales existentes en el campo, sumado al poco prestigio del trabajo agrícola, son factores que van contra la estabilidad de la fuerza de trabajo en las UBPC.

Las limitaciones estructurales en el funcionamiento de las UBPC abarcan, ante todo, la ausencia en los niveles tanto meso como macro-económicos de cambios institucionales significativos en el papel de los órganos estatales (Rodríguez, 1999). No se ha avanzado mucho en la transformación del Estado de agente productor principal y administrador central, en regulador de los procesos económicos; los cambios dados en el sector agrario han sido más formales que reales. Así, el Estado ha continuado ejerciendo un excesivo control, a veces directo e indirecto, en la producción, compra y comercialización, así como en la asignación de los insumos, además de mantener un virtual monopolio, o cuando menos, una posición dominante en estas actividades.

Asimismo, el Estado adquiere a bajos precios los productos de las UBPC, mientras les vende los suministros e insumos para la producción, así como los servicios, a precios elevados. Ya es evidente que estos mecanismos se han tornado en un freno al incremento de la productividad del trabajo (González, 1998). De aquí que un objetivo original en la creación de las UBPC, como era el de incentivar el trabajo y así aumentar la productividad, ha sido socavado por las desproporciones de dichas relaciones. De esta forma, la conexión entre la productividad y los ingresos no aparece debido a la monopolización estatal de los precios: sin estímulo material no hay interés en el aumento de la producción. 
Otro aspecto que incide en las trabas estructurales que afectan a las UBPC tiene que ver con las medidas que tienden a fortalecer la infraestructura y medios de transporte que faciliten el acceso de las cooperativas al mercado agropecuario. No obstante, la autorización para que estas unidades adquieran las producciones de productores independientes o cooperativas de consumo y servicios les abren nuevas posibilidades para incrementar sus fuentes de ingresos por la vía de la comercialización, al tiempo que limitan la presencia de intermediarios parasitarios en el mercado.

Otro obstáculo estructural para el buen funcionamiento de las UBPC tiene que ver con el sistema de asignación de los insumos. Más de un observador ha llamado la atención sobre la ausencia de mercados desregulados para los bienes intermedios industriales y medios básicos, destacando el papel que ejerce el Estado de virtual monopolio en los abastecimientos (Bu Wong, 1996). Se reconoce por amplios círculos de especialistas y en eventos académicos, que este proceso de asignación de recursos no corresponde a la diversificación experimentada en las formas de explotación, ni a la multiplicación de los agentes económicos ni a la autonomía que, al menos, en las disposiciones oficiales se le han otorgado a las UBPC.

Por último, otra cuestión presente en las desviaciones estructurales producidas en el trabajo de las UBPC corresponde a la ausencia de representación para la canalización y defensa de sus intereses. Los miembros de las cooperativas son dueños de las condiciones de producción (excepto la tierra) y, sin embargo, están organizados como asalariados pertenecientes al Sindicato de Trabajadores Agropecuarios y Forestales, mientras que, por otra parte, están subordinados a los Ministerios de la Agricultura y del Azúcar. La condición del cooperativistapropietario-proletario y su subordinación a instancias estatales, no posibilita el desarrollo de acciones colectivas para la defensa de sus intereses, no disponiendo de asociaciones representativas independientes.

\section{EL COOPERATIVISMO CUBANO: FUNDAMENTO PARA UN DESARROLLO SOSTENIBLE}

Hoy día, aún no puede afirmarse que las transformaciones ocurridas en el sector agropecuario y el tipo de economía mixta que parece consolidarse, hayan dado respuesta adecuada y eficiente a los problemas históricos de la agricultura cubana: la escasez de mano de obra, la autosuficiencia alimentaria y la disminución de la dependencia externa para la alimentación de la población.

Sin embargo, teniendo en cuenta que el nuevo cooperativismo cubano nació en momentos de una profunda crisis económica, y que su actividad tiene que desenvolverse dentro de esa crisis, hay que reconocer que la consolidación y logros parciales de las UBPC durante casi una década es un mérito a 


\section{RIS}

RETISTA INTERNACIONAL DE SOCIOLOGIA

N" 28. Enero-Abril, 2001

HANS-JÜRGEN BURCHARDT

(CPA) constituidas a partir de 1976 mediante la unión de los medios productivos, incluida la tierra, por grupos de productores privados.

A la vez, un punto poco debatido en torno al funcionamiento de las UBPC ha sido el de su autonomía política; es criterio del autor, que la autogestión de las cooperativas y su conducción democrática son razones decisivas para el éxito del modelo encarnado en las UBPC. Cuando a los dueños colectivos les falta su propia autonomía y se les limita la cogestión democrática, entonces no funciona el cooperativismo. Parece recomendable que las funciones del Estado sean menos intervencionistas, se otorguen seguridades legales más amplias y transparentes, así como se abran espacios para crear estructuras propias y más diversificadas de organización; todo lo cual desembocaría en una amplia cultura pública del cooperativismo. La clave estaría aquí en el punto de equilibrio entre las aspiraciones y las potencialidades de las cooperativas para desarrollarse. Así, un excesivo tutelaje castraría las bases de su consolidación y retardaría innecesariamente su protagonismo económico y social; mientras que, una excesiva autonomía, conduciría al desorden y desvirtuaría los principios del cooperativismo.

Al mismo tiempo, al fluctuar los conflictos latentes entre los intereses particulares de los grupos productivos y las responsabilidades compartidas frente a la sociedad, generados por el nuevo proceso cooperativista, no se deben potenciarse solo los criterios de eficiencia económica. También tienen que ser tomados en cuenta los promovidos políticamente en la sociedad, para que sea propagada "...la autogestión como una vía alternativa de socialización" (Valdés, 1997: 203).

Tampoco debe olvidarse que las UBPC desarrollan sus actividades dentro de un marco local y regional. Así, una mayor racionalidad en el tamaño de las cooperativas, la diversificación de los cultivos, las posibilidades de estructurar redes de comercialización directa a la población en el ámbito local, la autorización para la prestación de servicios, junto con la vinculación horizontal entre las cooperativas y otras empresas locales, facilitaría la integración de las UBPC en la política y estrategias de los gobiernos municipales.

Por esta vía surgirían efectos sinérgicos entre las unidades colectivas y el desarrollo local. Así, podrían crecer nuevas local economies, que tendrían la oportunidad de ser no sólo el fundamento de una economía sostenible, sino, también, la célula de una nueva socialización de la sociedad cubana, lo que, como efecto positivo inmediato, reforzaría, a la vez, la legitimidad social y política del régimen cubano (Burchardt, 1999, Dilla, 1996).

De lo apuntado se desprende que el proceso cooperativo engendrado por las UBPC podría convertirse en un polo que impulse otras formas organizativas dentro de la reforma empresarial en marcha. No resulta un secreto afirmar que el carácter que asuman los cambios en la esfera de la dirección de la economía marcarán su impronta en el entramado político y social de la Isla. Entre las 
transformaciones introducidas en las empresas que aspiren a una autonomía real, a la elevación de la productividad y a un compromiso social, parece de interés promover, como una de sus variantes, las cooperativas. Un efectivo modelo cooperativista contribuiría a difundir y fortalecer una distribución social más justa, así como a una democratización de la economía y de la sociedad.

Muchas son las ventajas que aportaría el desarrollo exitoso del proceso de cooperativización en el agro. En primer lugar, asegurar una base de abastecimiento de productos alimenticios estables y a precios accesibles a la gran mayoría de la población, tendría positivos efectos psicológicos sobre los consumidores. A la vez, mejores salarios y condiciones de trabajo en la agricultura podrían absorber una masa importante de desempleados. Esto tendría repercusión evidente e impactos directos más dinámicos en las regiones y niveles locales, sin olvidar que, una ampliación de las formas cooperativas, mayores descentralizaciones, así como la creación de nuevas unidades campesinas serían una respuesta positiva para "recampesinar" el agro; y contribuir a detener la emigración del campo a la ciudad (Valdés, 1997).

La cooperativización generada por la formación de las UBPC creó una amplificación de la economía autogestionada sobre bases colectivas en un grupo numeroso de la población. Así, los "ubepecistas" representan algo más del 10\% de la fuerza de trabajo ocupada y, en su conjunto, influyen aproximadamente en la cuarta parte de los habitantes del país. Si este sector de la economía se convierte en próspero y altamente socializado, formaría en la sociedad cubana un influyente grupo que no podría ser ignorado. La consolidación de una capa de productores ligados directamente a la producción material conformaría una barrera a la imposición de intereses políticos particulares de minorías influyentes, así como tendería a equilibrar las relaciones entre el campo y la ciudad (Burchardt, 1999).

En resumen, puede afirmarse que un posible itinerario de los cambios operados en el sector agropecuario cubano, y los que son factibles de prever para un próximo futuro, reconocería que: "Sin la reanimación de la agricultura de consumo interno y del sector agroexportador y su rentabilización, no puede hablarse de superación de la crisis económica ni de saneamiento real de las finanzas internas... Los objetivos básicos de esta reforma son la diversificación de las formas económicas de producción y de los agentes productivos, el redimensionamiento de las entidades productivas, la introducción y generalización de la agricultura de bajos insumos y alta densidad de trabajo, la apertura del mercado y la generación de nuevos incentivos capaces de estabilizar y recapturar la fuerza de trabajo necesaria para este sector. La autonomía y la autogestión financiera y participativa son claves para la racionalización y el logro de la rentabilidad de la agricultura nacional. Esta es, definitivamente, la vía magistral para dar solución a los dos cuellos de botella fundamentales que aflijen al país: el problema alimentario y la escasez de divisas" (Figueroa, 1996: 18-19). 
RIS

RETISTA INTERNACIONAL DE SOCIOLOCI.

N" 28. Enero-Abril. 2001

HANS-JÜRGEN BURCHARDT

Por otra parte, algunos estudiosos de la realidad cubana consideran que las transformaciones introducidas en el funcionamiento del sector agropecuario en Cuba pueden calificarse de una "Tercera Reforma Agraria". Aún cuando la 'economía mixta', que apreciamos hoy en los tipos y formas de explotación agraria en Cuba, da lugar a valoraciones de este alcance, también existen cuestionamientos que no pueden pasarse por alto. Ante todo, señalaríamos el control directo e indirecto que el Estado ejerce sobre el sector, así como la ausencia de institucionalizaciones claras y precisas para validar legalmente los cambios introducidos, sin olvidar la falta de promulgación de reglas transparentes para el usufructo de la tierra, la tenencia de explotaciones especializadas (tabaco, café, cacao, etc.), así como las posibilidades de hacer reversibles las medidas implementadas.

Finalmente, un análisis dentro de la problemática del sector agropecuario en Cuba y el papel que en ella desempeñan las UBPC, no puede desconocer los peligros presentes en la degradación del entorno agrario. Hoy, 11 de las 14 provincias de la Isla evidencian síndromes propios de zonas desérticas: el 14\% de las tierras están afectadas por salinidad y sodisidad; más de un millón 500 mil has corresponden a suelos áridos, semi-áridos y subhúmedos secos; en el $29 \%$ está presente la erosión; el $37 \%$ muestra deficiente drenaje; el $41 \%$ baja fertilidad, y un $64 \%$ muestra insuficiente contenido de materia orgánica. Las plantas leñosas indeseables cubren más de un millón 300 mil ha (Díaz, 1997). Estos graves efectos ecológicos constituyen un desafio insoslayable para la consecución de una economía agraria sustentable.

Por último, para alcanzar las potencialidades actuales contenidas en la política agraria de la Isla es necesaria una reorientación radical. El cooperativismo cubano hoy en día no es un reto de factibilidad, sino, sobre todo, una cuestión de voluntad política. Quien quiera para la Cuba del nuevo siglo una vinculación de la eficiencia económica con un sistema que priorice al hombre y su entorno social, la defensa del medio ecológico y las relaciones de propiedad con presencia de una amplia cogestión participativa, ha de promover y hacer de la llamada "Tercera Reforma Agraria" una vía irreversible.

\section{BIBLIOGRAFÍA}

ARANDA, S. (1968), La revolución agraria en Cuba, México.

BU WONG, A. (1996), "Las UBPC y su necesario perfeccionamiento", Cuba: Investigación Económica, $\mathrm{n}^{\circ} 2$, abril-junio, pp. 15-43.

BRUS, W. y K. LASKI (1989), From Marx to the market: socialism in search of an economic system, Oxford. 
BURCHARDT, H. (1995), “La economia sumergida: ¿de la ilegalidad al programa?”, Karl Kohut y Günter Mertins (eds.), Cuba en 1995: un diálogo entre investigadores alemanes y cubanos, Augsburg, pp. 13-19, Arbeitshefte des ISLA.

(1996), Kuba: Der lange Abschied von einem Mythos, Stuttgart.

(1999), Kuba: Im Herbst des Patriarchen, Stuttgart.

CARRANZA VALDÉS, J., L. URDANETA GUTIÉRREZ y P. MONREAL GONZÁLEZ (1995), Cuba: la restructuración de la economia - una propuesta para el debate, La Habana.

CARRIAZO MORENO, G. (1994), "Cambios estructurales en la agricultura cubana: la cooperativización”, Economía Cubana - Boletin Informativo, n 18, pp. 14-29.

COLECTIVO DE AUTORES (1995), La Reforma Económica Cubana, La Habana.

DEERE, C.D. (1994), "Implicaciones agricolas del comercio cubano", Economía Cubana Boletín Informativo, $\mathrm{n}^{\circ} 18, \mathrm{pp} .3-14$.

DÍAZ VÁZQUEZ, J.A. (1997), Cuba: Reforma económica dentro del socialismo, Centre de Investigación de Economía Internacional, La Habana.

DILLA, H. (ed.). (1996), La participación en Cuba, La Habana.

EER (Equipo de Estudios Rurales) (eds.) (1996), UBPC-desarrollo rural y participación, La Habana.

EER (Equipo de Estudios Rurales) (eds.) (1998), UBPC-desarrollo rural y participación social, La Habana.

FIGUERAS, M. (1994), Aspectos estructurales de la economia cubana, La Habana.

FIGUEROA, V. (1996), "El nuevo modelo agrario en Cuba bajo los marcos de la reforma económica", Equipo de Estudios Rurales (EER). UBPC - desarrollo rural y participación, La Habana, pp. 1-45.

GACETA OFICIAL DE LA REPÚBLICA DE CUBA (1993), Decreto-Ley 142 del Consejo de Estado. Sobre las unidades básicas de producción cooperativa, no. 6. 21.9.

GONZÁLEZ GUTIÉRREZ, A. (1995), "La economía sumergida en Cuba”, Cuba: Investigación Económica, no. 2, pp. 77-101.

(1998), "Economía y sociedad: los retos del modelo", Temas, no. 11, pp. 4-29.

MESA-LAGO, C. (1995), "Evaluavión y perspectivas de la reforma económica cubana", Hoffmann (ed.), Cuba: apertura y reforma económica, Caracas, pp. 59-89.

MILlARES, M. (1999), "Se liquidará el Presupuesto del 1999 con un déficit menor al aprobado", Granma, 30-12-1999. 
RIS

REIISTA INTERNACIONAL DE SOCIOLOGIA

N" 28. Enero-Abril. 2001

NOVA GONZÁLEZ, A. (1996), "El mercado agropecuario cubano: práctica y teoria", Economia y Desarrollo, no 3-4, pp. 41-50.

(1997), Economia Agropecuaria, Ministerio de Economia y Planificación, La Habana.

PINO SANTOS, O. (1999), "La Ley de la reforma agraria de 1959 y el fin de las oligarquías en Cuba", Temas, n 16-17, pp. 42-60.

RODRÍGUEZ CASTELLÓN, S. (1999), "La evolución del sector agropecuario en los noventa, en Balance de la economía cubana a finales de los 90s", Informe anual del Centro de Estudios de la Economía Cubana, La Habana, pp. 61-81.

VALDÉS, O. (1990), La socialización de la tierra en Cuba, La Habana.

VALdÉS PAZ, J. (1997), Procesos agrarios en Cuba 1959-1995, La Habana. 